\title{
The transformative journey of chronic myeloid leukemia
}

\author{
Pratap Neelakantan ${ }^{1,2}$ \& Adam J Mead*,2,3 \\ ${ }^{1}$ Department of Haematology, Royal Berkshire Hospital, Reading, UK \\ ${ }^{2} \mathrm{NIHR}$ Biomedical Research Centre, University of Oxford, Oxford, UK \\ ${ }^{3}$ Medical Research Council Molecular Haematology Unit, WIMM, University of Oxford, Oxford, UK \\ *Author for correspondence: Tel..+44 1865222 489; adam.mead@imm.ox.ac.uk \\ $\mathbf{c}_{\text {it }}$ is hard to believe that one of the key goals of therapy is now to achieve deep molecular \\ remission so that treatment-free remission and 'cure' might become a reality"
}

First draft submitted: 20 April 2018; Accepted for publication: 20 July 2017; Published online: 28 August 2018

Five thousand years after the ancient physicians began conceiving treatment methodologies for cancers in the form of arsenic, the publication of the results of the pivotal IRIS trial with imatinib for chronic myeloid leukemia (CML) in 2003 was a remarkable landmark [1]. Imatinib, one of the first examples of a so called 'magic-bullet' therapy, transformed the outlook of a cancer which, up to that point, had a median survival of 3-5 years from diagnosis [2]. Indeed, since CML was first described in 1845 by JH Bennett in Edinburgh, and the causative genetic lesion of CML, the Philadelphia $(\mathrm{Ph})$ chromosome, was discovered 140 years later by J Rowley, this disease has been at the forefront of advances in diagnosis and treatment of cancer. Most patients between the years 1970-2000 needed bone marrow transplantation to offer any realistic hope of long-term survival and this was a procedure restricted to a minority of patients and also associated with significant toxicity. The discovery of the Ph chromosome fast tracked the pace of discoveries in the field, to the extent that the first targeted therapy for this disease was being tested in first-in-man trials during the late 1990s, culminating in the remarkable results of the IRIS trial [1]. For the first time, patients were achieving extremely high rates of sustained cytogenetic remissions [1].

Fast forward 10 years and newer '2nd generation' tyrosine kinase inhibitors (TKI) were further transforming the outlook for patients with CML. These newer 2nd generation TKIs were able to induce faster and deeper responses and also offered the minority of patients who fail imatinib an alternative treatment [3]. Indeed, such is the success of the current therapeutic armamentarium, that recent studies have shown that the life expectancy for CML patients is now approaching that of the normal population, a remarkable turnaround over a relatively short period of time [2]. The goals of therapy in CML have consequently been completely rewritten and are now focused on the achievement of faster and deeper molecular responses which correlate with better outcomes for patients.

Although TKIs are highly-effective treatments, until recently the conventional wisdom was that therapy needed to be 'lifelong', an important consideration as TKIs are not without side effects. The next chapter in the CML story started with publication of the French STIM study, which suggested that it was possible to successfully stop TKI therapy without disease recurrence in approximately half the patients who achieve deep molecular responses [4]. Subsequently, large prospective clinical trials of the 2 nd generation TKI nilotinib have confirmed these findings, leading to this drug receiving the first licence for 'treatment-free remission' [5,6]. Today, it is hard to believe that one of the key goals of therapy is now to achieve deep molecular remission so that treatment-free remission and 'cure' might become a reality for many patients in routine clinical practice. Although hurdles remain, in particular to increase the proportion of patients who achieve such deep molecular remissions, if the CML story continues along its previous trajectory, we are well on the way toward achieving the dream of cure for the majority of patients with CML. 
financial involvement with any organization or entity with a financial interest in or financial conflict with the subject matter or materials discussed in the manuscript apart from those disclosed.

No writing assistance was utilized in the production of this manuscript.

Open access

This work is licensed under the Creative Commons Attribution 4.0 License. To view a copy of this license, visit http://creativecommons.org/licenses/by/4.0/

\section{References}

1. O'Brien SG, Guilhot F, Larson RA et al. Imatinib compared with interferon and low-dose cytarabine for newly diagnosed chronic-phase chronic myeloid leukemia. N. Engl. J. Med. 348, 11 (2003).

2. DeAngelo DJ, Ritz J. Imatinib therapy for patients with chronic myelogenous leukemia: are patients living longer? Clin. Cancer Res. 10, 1-3 (2004).

3. Abruzzese E, Breccia M, Latagliata R. Second-generation tyrosine kinase inhibitors in first-line treatment of chronic myeloid leukemia (CML). BioDrugs 28, 17-26 (2014).

4. Mahon F, Rea D, Guilhot J et al. Discontinuation of imatinib in patients with chronic myeloid leukemia who have maintained complete molecular remission for at least 2 years: the prospective, multicentre Stop Imatinib (STIM) trial. Lancet Oncol. 11, 1029-1035 (2010).

5. Hochhaus A, Masszi T, Giles FJ et al. Treatment-free remission following frontline nilotinib in patients with chronic myeloid leukemia in chronic phase: results from the ENESTfreedom study. Leukemia 31, 1525-1531 (2017).

6. Hughes TP, C Boquimpani, DW Kim, N Benyamaini, NC Clementino, V Shuvaev.Treatment-free remission (TFR) in patients (pts) with chronic myeloid leukemia in chronic phase (CML-CP) treated with second-line nilotinib (NIL): First results from the ENESTop study. American Society of Clinical Oncology (ASCO) Annual Meeting, ILUSA, 3-7 June, 2016 (Poster Presentation: Abstract \#7054). 